\title{
Hermitian-Yang-Mills connections and beyond
}

\author{
Jun Li
}

\begin{abstract}
This is a survey article on Donaldson-Uhlenbeck-Yau theorem and some of its further developments.
\end{abstract}

\section{Introduction}

The work of Yang and Mills on a non-abelian version of the electromagnetic Lagrangian formed the seed of one of the greatest triumphs of the twentieth century physics, the standard model. Its analogue over a Riemannian four-manifold $(M, g)$ rests on the Yang-Mills functional

$$
Y M(A)=\int_{M}\left|F_{A}\right|^{2}
$$

where $F_{A}$ are the curvature tensors of hermitian connections $A$ on hermitian vector bundles $E$ (for the structure group $G=U(r)$ ). Yang-Mills connections are connections satisfying the Euler-Lagrangian equations

$$
d_{A}^{*} F_{A}=d_{A} F_{A}=0 .
$$

There is a class of connections that attain the minimum of the YangMills functional: the Anti-Self-Dual (in short ASD) connections, connections whose curvature tensors $F_{A}$ are ASD:

$$
*_{g} F_{A}=-F_{A} .
$$

When the underlying manifold $M$ are two-dimensional complex manifolds (i.e. $\operatorname{dim}_{\mathbb{R}} M=4$ ) and the Riemannian metrics $g$ are induced by Kahler forms $\omega$ on $M, U(r)$ ASD connections, viewed as hermitian connections on the associated rank $r$ hermitian vector bundles $E$, are hermitian connections of integrable complex structures on $E$ satisfying an additional

2010 Mathematics Subject Classification. 14D20, 14D22, 14D24, 53C07.

Key words and phrases. Hermitian-Yang-Mills, stable bundles, Donaldson-YauUhlenbeck.

The author was partially supported by an NSF grant NSF-1104553. 
$\omega$-traceless type conditions. The notion of ASD connections for Kahler manifolds later was generalized to that of Hermitian-Yang-Mills (in short HYM) connections. Kobayashi showed that the holomorphic vector bundle $(E, \bar{\partial})$ induced by an irreducible HYM connection on $(E, h)$ is necessarily MumfordTakemoto stable [15], a notion introduced earlier in algebraic geometry. Relying on the foundational work of Uhlenbeck on the removable singularity for Yang-Mills connections on four-manifolds [32, 33], Donaldson showed that a Mumford-Takemoto stable holomorphic structure $\bar{\partial}$ on a vector bundle $E$ over an algebraic surface admits an irreducible Hermitian-Yang-Mills connection $[\mathbf{8}]$.

Later, Uhlenbeck-Yau generalized this theorem to the existence of irreducible Hermitian-Yang-Mills connections on slope stable holomorphic vector bundles on compact Kahler manifolds of arbitrary dimensions [34]. This established the DUY (Donaldson-Uhlenbeck-Yau) correspondence that a holomorphic bundle on a compact Kahler manifold admits an irreducible HYM metric if and only if it is slope stable.

In the remainder of this article, we will briefly recall this theorem, this correspondence, and its generalizations.

\section{Hermitian-Yang-Mills connections}

Let $(X, \omega)$ be a Kahler manifold. The space of Anti-Self-Dual 2-forms $\Omega^{2,-}$ is a pointwise orthogonal complement of $\omega$ in the space of real $(1,1)$ forms $\Omega^{1,1}$. Thus a connection $A$ on a vector bundle is ASD if its curvature $F_{A}$ obeys

$$
\Lambda F_{A}=F_{A}^{2,0}=F_{A}^{0,2}=0,
$$

where $\Lambda$ is the contraction with respect to $\omega$ :

$$
\Lambda F_{A}=\left(F_{A} \wedge \omega^{\operatorname{dim} X-1}\right) / \omega^{\operatorname{dim} X} .
$$

This leads to the notion of HYM connections. Let $(E, h)$ be a Hermitian vector bundle on a Kahler manifold $(X, \omega)$. A hermitian connection $A$ on $(E, h)$ is called HYM if

$$
\Lambda F_{A}=2 \pi i \mu I_{E}, \quad F_{A}^{2,0}=F_{A}^{0,2}=0, \quad \mu \in \mathbb{R} .
$$

Note that $F_{A}^{0,2}=\left(\bar{\partial}_{A}\right)^{2}$, thus (2) implies that $\bar{\partial}_{A}$ is integrable.

The constant $\mu$ is a topological invariant. By Chern-Weil theory,

$$
d(E, \omega)=\int_{X} c_{1}(E) \wedge \omega^{\operatorname{dim} X-1}=\int_{X} \frac{i}{2 \pi} \operatorname{Tr} F_{A} \wedge \omega^{\operatorname{dim} X-1} .
$$

Integrating the first identity in (2), the constant $\mu$ takes the form:

$$
n ! \mu \cdot \operatorname{Vol}_{X}=-\mu(E, \omega), \quad \mu(E, \omega)=\frac{d(E, \omega)}{\operatorname{rank} E} .
$$

From (1) and (2), we see that over Kahler surfaces degree zero HYM connections are the same as ASD connections. 
HYM connections lead naturally to Mumford-Takemoto stable holomorphic vector bundles. A holomorphic vector bundle $E$ is Mumford-Takemoto stable (resp. semistable) if for every subsheaf $F \neq 0$ of $E$ with $\operatorname{rank} F<$ $\operatorname{rank} E$, we have

$$
\mu(F, \omega)<\mu(E, \omega), \quad(\text { resp. } \leq) .
$$

(For any sheaf $F, d(F, \omega)=\int c_{1}(F) \wedge \omega^{\operatorname{dim} X-1}$.) Because this stability is on the slope decreasing property after passing to subsheaves of smaller ranks, it is also called slope-stable, and sometimes, $\mu$-stable.

Over smooth curves, HYM connections (of degree 0) leading to MumfordTakemoto stable bundle is a consequence of a theorem of NarasimhanSeshadri [24]. For Kahler manifolds, it is proved by Kobayashi [15].

This can be seen using the Chern-Weil forms of the Chern classes of vector bundles. Since this argument is used in Uhlenbeck-Yau's proof of the existence of HYM connections, we will outline the argument.

Given a connection $A$ on $E$, the splitting of forms decomposes the connection

$$
D_{A}=\partial_{A} \oplus \bar{\partial}_{A}: \Omega^{0}(E) \longrightarrow \Omega^{1,0}(E) \oplus \Omega^{0,1}(E)
$$

and the curvature

$$
F_{A}^{2,0}=\partial_{A} \circ \partial_{A}, \quad F^{0,2}=\bar{\partial}_{A} \circ \bar{\partial}_{A}, \quad \text { and } \quad F_{A}^{1,1}=\partial_{A} \circ \bar{\partial}_{A}+\bar{\partial}_{A} \circ \partial_{A} .
$$

Suppose $A$ is a HYM connection. Then $F_{A}^{0,2}=\bar{\partial}_{A} \circ \bar{\partial}_{A}=0$, which is the integrability condition for the operator $\bar{\partial}_{A}$, or that $\left(E, \bar{\partial}_{A}\right)$ is a holomorphic vector bundle.

The holomorphic bundle $\left(E, \bar{\partial}_{A}\right)$ is slope stable when $A$ is an irreducible HYM. To see this, we let $L \subset\left(E, \bar{\partial}_{A}\right)$ be a holomorphic subbundle. By using the Hermitian metric $h$, we can write $E$ as a direct sum $L \oplus L^{\perp}$, where $L^{\perp}$ is the orthogonal complement of $L \subset E$, a smooth subbundle of $E$. We next decompose the restriction of $D_{A}$ to $L$ as

$$
\left.D_{A}\right|_{L}=D_{L} \oplus B_{1}: \Omega^{0}(L) \longrightarrow \Omega^{1}(L) \oplus \Omega^{1}\left(L^{\perp}\right)=\Omega^{1}(E) .
$$

In this form, $D_{L}$ is a Hermitian connection of $\left(L,\left.h\right|_{L}\right)$, and $B_{1}$ is a tensor in $\Omega^{1,0}\left(L^{\vee} \otimes L^{\perp}\right)$. (It is a $(1,0)$-form since $L \subset E$ is holomorphic.) Similarly, by restricting $D_{A}$ to $L^{\perp} \subset E$, we get $\left.D_{A}\right|_{L^{\perp}}=D_{L^{\perp}} \oplus B_{2}$, with $D_{L^{\perp}}$ a Hermitian connection on $L^{\perp}$ and $B_{2}$ a tensor in $\Omega^{0,1}\left(\left(L^{\perp}\right)^{\vee} \otimes L\right)$.

There is a more intrinsic form of expressing $B_{1}$. We let

$$
\Pi: E \longrightarrow L \subset E
$$

be the projection via the smooth isomorphism $E \cong L \oplus L^{\perp}$. The projection $\Pi$ defines $L$ as the image bundle of $\Pi$. Since $L \subset E$ is a holomorphic subbundle, $\Pi^{\perp} \circ \bar{\partial} \Pi=0$. The other part,

$$
\Pi^{\perp} \circ \partial_{A} \Pi=B_{1},
$$

is the second fundamental form of $L \subset E$. 
From the above decomposition, we obtain, in the matrix form,

$$
D_{A}=\left(\begin{array}{cc}
D_{L} & B_{2} \\
B_{1} & D_{L^{\perp}}
\end{array}\right)
$$

and

$$
F_{A} \circ \Pi=\left[\begin{array}{cc}
\left(D_{L}\right)^{2}+B_{2} \wedge B_{1} & 0 \\
B_{1} \circ D_{L}+D_{L^{\perp}} \circ B_{1} & 0
\end{array}\right] .
$$

We now estimate the degree $d(L, \omega)$ using the Chern-Weil theory. First, (with $n=\operatorname{dim} X$,) we have

$$
d(L, \omega)=\int c_{1}(L) \wedge \omega^{n-1}=\int \frac{i}{2 \pi} \operatorname{Tr}\left(D_{L}\right)^{2} \wedge \omega^{n-1} .
$$

Combined with the expression of $F_{A} \circ \Pi$, we have

$$
\int \frac{i}{2 \pi} \operatorname{Tr}\left(D_{L}\right)^{2} \wedge \omega^{n-1}=\int \frac{i}{2 \pi} \operatorname{Tr}\left(\Lambda F_{A} \circ \Pi\right) \omega^{n}-\int \frac{i}{2 \pi} \operatorname{Tr}\left(B_{2} \wedge B_{1}\right) \wedge \omega^{n-1} .
$$

Using that $A$ is a hermitian connection,

$$
\int \frac{i}{2 \pi} \operatorname{Tr}\left(B_{2} \wedge B_{1}\right) \wedge \omega^{n-1}=\int \frac{1}{2 \pi}\left|\Pi^{\perp} \partial_{A} \Pi\right|^{2} \omega^{n} .
$$

This proves that

$$
\mu(L, \omega)=\frac{1}{\operatorname{rank} L}\left(\int \frac{i}{2 \pi} \operatorname{Tr}\left(\Lambda F_{A} \circ \Pi\right) \omega^{n}-\int \frac{1}{2 \pi}\left|\Pi^{\perp} \partial_{A} \Pi\right|^{2} \omega^{n}\right) .
$$

Note that this holds for any hermitian connection $A$. Assume $A$ is a HYM connection, then $\Lambda F_{A}=2 \pi i \mu \mathrm{I}_{E}$ and

$$
\operatorname{Tr}\left(\Lambda F_{A} \circ \Pi\right)=\mu(E, \omega) \cdot \operatorname{rank} L .
$$

Plugging into (2), we see that

$$
\mu(L, \omega) \leq \mu(E, \omega),
$$

and the strict inequality holds when $L^{\perp}$ is not a holomorphic subbundle, which holds true if $A$ is irreducible.

We remark that the same argument also works for $L \subset E$ a subsheaf. Since we can assume without lose of generality that away from a codimension two subset the sheaf $L$ is locally free and the inclusion $L \subset E$ is a vector sub-bundle, the preceding argument remains valid using the $L^{2}$-version of the Chern-Weil formula.

This proves that when $A$ is an irreducible HYM connection, then $\left(E, \bar{\partial}_{A}\right)$ is slope stable. By an induction on the rank of $E$, it also shows that the holomorphic bundle induced by a HYM connection is necessarily a polystable bundle. Here by a poly-stable vector bundle we mean that it is a direct sum of stable bundles of identical slopes. 


\section{Donaldson-Uhlenbeck-Yau theorem}

Donaldson-Uhlenbeck-Yau theorem is the correspondence of HYM connections and Mumford-Takemoto poly-stable holomorphic structures on vector bundles.

Let $(E, \bar{\partial})$ be a slope stable vector bundle on a Kahler manifold $(X, \omega)$. The Donaldson-Uhlenbeck-Yau theorem states that there is a hermitian metric $h$ on $(E, \bar{\partial})$ such that its hermitian connection $A$ satisfies the HYM equation

$$
\Lambda F_{A}=2 \pi i \mu I_{E} .
$$

The technical part of this theorem is a milestone in geometric analysis, demonstrating directly how the failure of the existence of HYM metrics on a holomorphic bundle is caused by the existence of the destabilizing subsheaf of the bundle.

Traditionally, one searches for a Yang-Mills connection by studying the Yang-Mills flow initiating from a base connection $A_{0}$ :

$$
\frac{\partial A}{\partial t}=-d_{A}^{*} F_{A}
$$

In case $E$ is a holomorphic vector bundle with $\bar{\partial}$ implicitly understood, which we assume throughout this section, we can choose $A_{0}$ to be a hermitian connection of $E$. Then the solutions to the Yang-Mills flow is a family of hermitian connections on $E$ (conforming to the given holomorphic structure).

Expressing $A$ as the hermitian connection of $h$, the Yang-Mills flow takes the form

$$
h^{-1} \frac{\partial h}{\partial t}=-2 i\left(\Lambda F(h)-2 \pi i \mu I_{E}\right)
$$

Here $F(h)$ is the curvature of the hermitian connection of $(E, \bar{\partial}, h)$.

Donaldson's insight is that these flows are gradient flows of an energy functional $V$ on the space of hermitian metrics. Assuming $X$ is an algebraic surface and the Kahler form $\omega$ is the first Chern form of an ample line bundle, Donaldson proved that $V$ is convex and bounded from below. (The proof used the restriction property of slope stable bundles to an ample divisor in an essential way.) The only critical points are HYM metrics (connection) up to constant scalings.

Applying the standard technique in PDE associated to gradient flows of a convex bounded energy functional, Donaldson showed that the solution $h_{t}$ to the parabolic system exists for all $t$. Also, when $h_{t}$ converges to $h_{\infty}$, that $V$ is bounded and $h_{t}$ is its gradient flow infers that $h_{\infty}$ is a HYM metric (connection).

To investigate the limiting behavior of $h_{t}$ when $h_{t}$ diverges, Donaldson used Uhlenbeck's weak $L^{p}$ compactness of connections and removable singularity theorem. Because of the parabolic system $h_{t}$ satisfies, the curvature $F\left(h_{t}\right)$ is bounded and the primitive part of $F\left(h_{t}\right)$, which is $\Lambda F(h)-2 \pi i \mu I_{E}$, 
tends to zero. Using the weak limit of the hermitian connections $A_{t}$, Donaldson constructed a HYM connection $A^{\prime}$ on a possibly different vector bundle $E^{\prime}$ of slope identical to that of $E$, and a non-trivial holomorphic map between them. Since $E$ is slope stable and $\left(E^{\prime}, \bar{\partial}_{A^{\prime}}\right)$ is semistable, this is possible only if $E \cong E^{\prime}$. This proves that $h_{t}$ converges to $h_{\infty}$.

TheOREM 3.1 (Donaldson [8]). A slope stable holomorphic vector bundle on a projective surface admits a unique (up to scaling) HYM connection.

This theorem is generalized by Uhlenbeck-Yau to general Kahler manifolds.

Theorem 3.2 (Uhlenbeck-Yau [34]). A slope stable holomorphic vector bundle on a smooth Kahler manifold admits a unique (up to scaling) HYM connection.

Uhlenbeck-Yau proved the existence of HYM connections by using continuity argument. They introduced a family of elliptic systems $L_{\epsilon}=0$ on hermitian metrics; it has the property that solutions to $L_{0}=0$ are HYM connections; solutions to $L_{\epsilon}=0$ exist for all $\epsilon>0$. They also proved that when the logarithms of the solutions $h_{\epsilon}$ (to $L_{\epsilon}=0$ ) have uniform $L^{2}$ bounds, then $h_{\epsilon}$ converges to a HYM connection as $\epsilon \rightarrow 0$. When the supremum norm of $h_{\epsilon}$ tends to infinity, they proved that the normalized $\rho_{\epsilon} h_{\epsilon}$ converges weakly to $h_{\infty}$, and the weak limit

$$
\Pi:=\lim _{s \rightarrow 0}\left(\mathrm{I}_{E}-h_{\infty}^{s}\right)
$$

exists, is a projection, and its image is a holomorphic destabilizing subsheaf of $E$.

Uhlenbeck-Yau's proof inspired a new thinking for other geometric problems in complex geometry. To familiar the readers with their proof, we outline it briefly here, focusing on the derivation of the destabilizing subsheaves via the failure of the convergence of $h_{\epsilon}$.

We fix a holomorphic vector bundle $E$; fix a base hermitian metric $\langle\cdot, \cdot\rangle$ on it. Then any other hermitian metric is given by a positive definite hermitian symmetric matrix $h$ via

$$
\langle\cdot, \cdot\rangle_{h}=\langle h \cdot, \cdot\rangle \text {. }
$$

Let $A_{0}$ be the hermitian connection of the base hermitian metric, the hermitian connection of $h$ is

$$
A_{h}=A_{0}+h^{-1} \partial_{0} h
$$

the HYM equation for $h$ (if $\mu=0$ ) is

$$
\Lambda F_{0}-\Lambda \bar{\partial}\left(h^{-1} \partial_{0} h\right)=0 .
$$

The system Uhlenbeck-Yau considered is

$$
L_{\epsilon}(h):=\Lambda F_{0}-\Lambda \bar{\partial}\left(h^{-1} \partial_{0} h\right)+\epsilon \log h=0 .
$$


(Since $h$ is hermitian symmetric positive definite, $\log h$ is well-defined.) The first part of Uhlenbeck-Yau's proof is to show that the system is solvable for all $\epsilon>0$.

To prove this, Uhlenbeck-Yau used a secondary continuity method by further introducing

$$
L_{\epsilon, \sigma}(h):=\Lambda F_{0}-\Lambda \bar{\partial}\left(h^{-1} \partial_{0} h\right)+\epsilon \log h-\sigma h^{-1 / 2} \Lambda F_{0} h^{1 / 2}=0 .
$$

This system has the trivial solution $h=\mathrm{id}$ for $\sigma=1$. Let

$$
T_{\epsilon}=\{\sigma \in[0,1] \mid \text { the system (6) is solvable }\} \text {; }
$$

it contains $\sigma=1$, thus is non-empty.

The set $T_{\epsilon}$ is both open and closed. The openness of $T_{\epsilon}$ follows from the invertibility of the linearization $\delta L_{\epsilon, \sigma}$. The closedness follows from an a priori estimate of the solutions $h_{\epsilon, \sigma}$ (to $L_{\epsilon, \sigma}=0$ ). For $\epsilon$ sufficiently large, Uhlenbeck-Yau developed a set of elaborate estimates sufficient for both the openness and closeness of $T_{\epsilon}$.

Knowing that $L_{\epsilon}=0$ is solvable for $\epsilon$ sufficiently large, refining their estimate, they went on to show that it is solvable for all $\epsilon>0$.

At this stage, we pause for a moment and ask what could happen when $E$ is not slope stable. In this case, the prior argument provides us a family $h_{\epsilon}$ for all $\epsilon>0$ that solves (5). Since $E$ has no HYM metric, the family $h_{\epsilon}$ must diverge.

The family $h_{\epsilon}$ diverges in a good way. An easy inspection of the system (5) shows that we can arrange $\operatorname{det} h_{\epsilon}=1$ for all $\epsilon$. Also, an estimate developed by Uhlenbeck-Yau guarantees that the $L^{2}$ norm and the $L^{\infty}$ norm of $\log h_{\epsilon}$ are equivalent. Suppose $h_{\epsilon}$ diverges, Uhlenbeck-Yau showed that the normalized family $\rho_{\epsilon} h_{\epsilon}$-normalized so that the supremum norm is one, converges weakly to a homomorphism $h_{\infty}$.

Uhlenbeck-Yau's insight is that $h_{\infty}$ is a homomorphism of $E$ whose kernel is a destabilizing subsheaf of $E$. Along this line, they defined

$$
\Pi:=\operatorname{id}_{E}-\lim _{s \rightarrow 0} h_{\infty}^{s} .
$$

They proved that $\Pi$ exists as a weak limit, that $\Pi$ is a projection almost everywhere, and as distribution

$$
\left|\left(\operatorname{id}_{E}-\Pi\right) \bar{\partial} \Pi\right|^{2}=0 .
$$

This shows that the "image sheaf" $L$ of $\Pi$ is "weakly" holomorphic.

After a technical tour de force, Uhlenbeck-Yau proved that $L$ is a holomorphic subsheaf of $E$. Because $\operatorname{det} h_{\epsilon}=1$ and $\rho_{\epsilon} \rightarrow \infty, h_{\infty}$ is nontrivial and $\operatorname{det} h_{\infty}=0$. Thus $L$ is a subsheaf of smaller rank.

It remains to show that this image sheaf $L \subset E$ is a destabilizing subsheaf of $E$. To prove this, we will mimic the subbundle case worked out earlier. Since $L$ is locally free away from a codimension two subset, we can use the $L^{2}$ Chern-Weil form of $c_{1}(L)$ to evaluate its degree. Like formula (4), we 
have

$\operatorname{deg} L=\int \frac{1}{2 \pi i} \operatorname{Tr} F\left(\left.h_{0}\right|_{L}\right) \wedge \omega^{n-1}=\int \frac{1}{2 \pi i} \operatorname{Tr}\left(F\left(h_{0}\right) \Pi\right) \wedge \omega^{n-1}-\int\left|\Pi^{\perp} \partial_{0} \Pi\right|^{2}$.

The term

$$
\begin{aligned}
\int \frac{1}{2 \pi i} \operatorname{Tr}\left(F\left(h_{0}\right) \Pi\right) \wedge \omega^{n-1} & =\int \frac{1}{2 \pi i} \operatorname{Tr}\left(\left(\Lambda F\left(h_{0}\right)+2 \pi i \mu I_{E}\right) \Pi\right) \cdot \omega^{n} \\
& =\int \frac{1}{2 \pi i} \operatorname{Tr}\left(\Lambda F\left(h_{0}\right)\right) \cdot \omega^{n}+\frac{\operatorname{rank} F}{\operatorname{rank} E} \operatorname{deg} E .
\end{aligned}
$$

By applying the system (5), Uhlenbeck-Yau proved that

$$
\int \frac{1}{2 \pi i} \operatorname{Tr}\left(\Lambda F\left(h_{0}\right)\right) \cdot \omega^{n} \geq \int\left|\Pi^{\perp} \partial_{0} \Pi\right|^{2} .
$$

This implies $\mu(L, \omega) \geq \mu(E, \omega)$, contradicting to $E$ being MumfordTakemoto stable.

"The most important development is the work of Uhlenbeck and Yau who have proved (this theorem) in full generality, $\cdots$ their proof is probably the most natural and display clearly the role of stability," commented by Donaldson [9, page 231]. In the same paper, he gave a simplified proof of Uhlenbeck-Yau't theorem for project manifolds.

\section{Further developments}

Along several directions, Uhlenbeck-Yau's theorem has been generalized. In the following we give an incomplete account of these developments.

The first direction is for more general complex manifolds. Buchdahl first considered the case where $M$ is a complex surface endowed with a hermitian metric whose hermitian form $\omega$ satisfies $\partial \bar{\partial} \omega=0[\mathbf{3}]$. Because $M$ is a surface and $\partial \bar{\partial} \omega=0$, we can use Chern forms $C_{1}(E, h)$ of any hermitian bundle $(E, h)$ to represent its first Chern class and define its $\omega$-degree

$$
\operatorname{deg} E=\int C_{1}(E, h) \wedge \omega .
$$

By the transgression formula for Chern forms, the degree is well defined. Using this, Buchdahl defined the notion of $\omega$-slope stable and proved the surface analogue of Donaldson's existence theorem for $\omega$-slope stable vector bundles. Shortly after, Li-Yau generalized Buchdahl's result to high dimensional hermitian manifolds $M$ with hermitian form $\omega$ satisfying $\partial \bar{\partial} \omega^{n-1}=0$, for $n=\operatorname{dim} M$ [19]. Adopting the technique of Uhlenbeck-Yau, they proved that Uhlenbeck-Yau theorem holds for this class of compact hermitian manifolds. We mention that the existence of HYM-connections on hermitian manifolds has been used to study class VII surfaces $[\mathbf{2 0}, \mathbf{2 1}, \mathbf{3 1}]$.

Donaldson-Uhlenbeck-Yau's theorem has also been generalized to slopestable reflexive coherent sheaves, by Bando-Siu [2]. They first introduced the notion of admissible metrics. An admissible metric on a torsion-free sheaf is a Hermitian metric on its locally free part such that its curvature $F$ is square integrable and the contraction $\Lambda F$ is uniformly bounded. They proved that 
any reflexive sheaf admits an admissible Hermitian metric, and any sequence of HYM metrics converges to an admissible HYM metric. Applying blowing up technique, they proved that any slope stable reflexive sheaves admits an admissible HYM metric.

Built upon an earlier work of Hitchin [13], Simpson studied Higgs bundle on a complex manifold $M[\mathbf{2 8}]$, which are pairs $(E, \theta)$ of holomorphic vector bundles $E$ and holomorphic maps (Higgs fields) $\theta: E \rightarrow E \otimes \Omega_{M}^{1}$, integrable in that $\theta \wedge \theta=0$. One defines the notion of slope stability by considering sub-Higgs sheaves $F \subset E$. The analogous of the $\bar{\partial}$-operator on a vector bundle is the mixed operator $D^{\prime \prime}=\bar{\partial}+\theta$; using a hermitian metric on $E$, one defines $\bar{\theta}$ and thus define $D^{\prime}=\partial+\bar{\theta}$. Finally, one defines the curvature to be $F(h)=D^{\prime} \circ D^{\prime \prime}+D^{\prime \prime} \circ D^{\prime}$, and call $h$ a HYM metric if $\Lambda F=\lambda \cdot$ id.

Adopting the proof of Uhlenbeck-Yau, Simpson proved that every slope stable Higgs bundle $E$ has a HYM metric. If $\theta \wedge \theta=0$, and $c_{1}(E)=0$ and $c_{2}(E) \cdot[\omega]^{n-2}=0$, then the connection is flat. One application of this existence theorem is to give a criterion for uniformization.

The proof of Uhlenbeck-Yau offers more than the existence of HYM metrics for stable objects. Over a Kahler manifold $M$, when $E$ is not slope stable, the non-convergence of the solutions $h_{\epsilon}$ (or rather the Yang-Mills flow $h_{t}$ ) gives rise to the Hardar-Narasimhan filtration of $E$. As $E$ is not slope stable, the direct sum of the graded pieces of the Hardar-Narasimhan filtration forms $\operatorname{gr}(E)$. Let $\operatorname{gr}(E)^{* *}$ be the double dual of $\operatorname{gr}(E)$, which is a direct sum of slope stable reflexive sheaves; let $Z^{\text {sing }}$ be the support of $\operatorname{gr}(E)^{* *} / \operatorname{gr}(E)$. Then the works of Daskalopoulos, Jacob, Råde, Sibley, and Wentworth $[\mathbf{5}, \mathbf{6}, \mathbf{1 4}, \mathbf{2 5}, \mathbf{2 6}, \mathbf{2 7}]$ shows that the Yang-Mills flow $h_{t}$, up to unitary gauge transformations, converges uniformly on every compact subset of $M-Z^{\text {sing }}$ to an admissible metric on $g r(E)^{* *}$ that is a direct sum of admissible HYM metrics on each stable factor of $g r(E)^{* *}$.

The last generalization we will mention is the existence of Supersymmetric String theory with torsion. This is a system introduced by Strominger [30] on a pair of hermitian metrics on the base complex manifold $M$ and an auxiliary complex vector bundle $E$ on $M$ satisfying $c_{1}(M)=c_{1}(E)=0$ and $c_{2}(M)=c_{2}(E)$. A trivial solution is when $M$ is Kahler and $E$ is slope stable, then the solution is given by the Calabi-Yau metric on $M$ and the HYM metric on $E$. When $M$ is not Kahler, the solution of this system consists of a balanced hermitian metric on $M$ plus a HYM metric on $E$ that satisfy a transgression on $c_{2}(M)=c_{2}(E)$. This system is a generalization of HYM metrics for not necessarily Kahler Calabi-Yau threefolds.

By a perturbation argument, Li-Yau produced a non-trivial solution on a class of Kahler Calabi-Yau threefolds [18]. By a direct existence argument, $\mathrm{Fu}-\mathrm{Yau}$ constructed the first example of non-trivial solutions of such a system over a non-Kahler Calabi-Yau base manifold [11]. 


\section{References}

[1] M. F. Atiyah and R. Bott, The Yang-Mills equations over Riemann surfaces, Philos. Trans. Roy. Soc. London Ser. A 308 (1982), 523-615.

[2] S. Bando and Y.-T. Siu, Stable sheaves and Einstein-Hermitian metrics, Geometry and analysis on complex manifolds, World Sci. Publishing (1994), 39-50.

[3] N. P. Buchdahl, Hermitian-Einstein connections and stable vector bundles over compact complex surfaces, Math. Ann. 280 (1988), 625-648.

[4] M.-T. Chuan, Existence of Hermitian-Yang-Mills metrics under conifold transitions, arXiv:1012.3107v1 [math.DG].

[5] G. D. Daskalopoulos, The topology of the space of stable bundles on a Riemann surface, J. Diff. Geom. 36 (1992), 699-746.

[6] G. D. Daskalopoulos and R. A. Wentworth, Convergence properties of the Yang-Mills flow on Kähler surfaces. J. Reine Angew. Math. 575 (2004), 69-99; and erratum in J. reine angew. Math. 606 (2007), 235-236.

[7] S. K. Donaldson, A new proof of a theorem of Narasimhan and Seshadri, J. Differential Geom. 18 (1983), no. 2, 269-277.

[8] S. K. Donaldson, Anti-self-dual Yang-Mills connections on complex algebraic surfaces and stable vector bundles, Proc. London Math. Soc. 3 (1985), 1-26.

[9] S. K. Donaldson, Infinite determinants, stable bundles and curvature, Duke Math. Jour. 54 (1987), 231-47.

[10] S. K. Donaldson and P.B. Kronheimer, The geometry of four-manifolds, The Clarendon Press, Oxford University Press, 1990.

[11] J. Fu and S.-T. Yau, The theory of superstring with flux on non-Kähler manifolds and the complex Monge-Ampère equation, J. Diff. Geom. 78(2008), 369-428.

[12] P. Gauduchon, Sur la 1-forme de torsion d'une variété hermitienne compacte, Math. Ann. 267(1984), 495-518.

[13] N. J. Hitchin, The self-duality equations on a Riemann surface, Proc. London Math. Soc. (3) 55 (1987), no. 1, 59-126.

[14] A. Jacob, The Yang-Mills flow and the Atiyah-Bott formula on compact Kähler manifolds, preprint, arXiv:1109.1550.

[15] S. Kobayashi, Differential geometry of holomorphic vetor bundles, Math. Seminar Notes (by I. Enoki) 41 (1982), Univ. of Tohyo.

[16] S. Kobayashi, Differential geometry of complex vector bundles, Princeton University Press, 1987.

[17] J. Li, Anti-Self-Dual connections and stable vector bundle, Gauge Theory and the Topology of Four-Manifolds, LAS/Park City Mathematics Series, Vol.4, 1998, 25-49.

[18] J. Li and S.-T. Yau, The existence of supersymmetric string theorey with torsion, J. Differential Geom. 70(2005), 143-181, MR2192064, Zbl 1102.53052.

[19] J. Li and S.-T. Yau, Hermitian-Yang-Mills connection on non-Kähler manifolds, Mathematical Aspects of String Theory, World Scientic, 1987, 560-573.

[20] J. Li, S.-T. Yau and F.Y. Zheng, On projectively flat Hermitian manifolds. Comm. Anal. Geom. 2 (1994), no. 1, 103-109.

[21] J. Li, S.-T. Yau and F.Y. Zheng, On projectively flat Hermitian manifolds. Comm. Anal. Geom. 2 (1994), no. 1, 103-109.

[22] M. Lübke, Stability of Einstein Hermitian vector bundles, Manuscripta Math. 42 (1983), no. 2-3, 245-257.

[23] H. Nakajima, Compactness of the moduli space of Yang-Mills connections in higher dimensions, J. Math. Soc. Japan 40 (1988), no. 3, 383-392.

[24] M.S. Narashiman and C.S. Seshadri, Stable and unitary vector bundles on compact Riemann surfaces, Ann. of Math. 82 (1965), 540-567.

[25] J. Råde, On the Yang-Mills heat equation in two and three dimensions, J. Reine. Angew. Math. 431 (1992), 123-163. 
[26] B. Sibley, Asymptotics of the Yang-Mills flow for holomorphic vector bundles over Kähler manifolds: The canonical structure of the limit, preprint, To appear in Crelle's Journal.

[27] B. Sibley and R. Wentworth, Analytic cycles, Bott-Chern forms, and singular sets for the Yang-Mills flow on Kähler manifolds, preprint, arXiv:1402.3808.

[28] C. T. Simpson, Constructing variations of Hodge structure using Yang-Mills theory and applications to uniformization, J. Amer. Math. Soc. 1(1988), 867-918.

[29] Y.-T. Siu, Lectures on Hermitian-Einstein metrics for stable bundles and KählerEinstein metrics, DMV Seminar, 8. Birkhäuser Verlag, Basel, 1987.

[30] A. Strominger, Superstings with Torsion, Nuclear Physics B, 274 (1986), no.2, 253284 .

[31] A. Teleman, Donaldson theory on non-Kählerian surfaces and class VII surfaces with $b_{2}=1$, Invent. Math. 162 (2005), no. 3, 493-521.

[32] K. K. Uhlenbeck, Removable singularities in Yang-Mills fields, Comm. Math. Phys., 83 no.1 (1982), 11-29.

[33] K. K. Uhlenbeck, Connections with $L^{p}$ bounds on curvature, Commun. Math. Phys. 83 (1982), 11-29.

[34] K. K. Uhlenbeck and S.-T. Yau, On the existence of hermitian Yang-Mills connections on stable bundles over compact Kähler manifolds, Commun. Pure Applied Math. 39 (1986), 257-93.

Department of Mathematics, Stanford University

E-mail address: jli@math.stanford.edu 